\title{
Production of Tin Single Crystals Foils from the Melt and Their Degrees of Perfection*
}

\author{
By Hideo Takaki**, Masashige Koyama***, Yoshikazu Tsujii*** \\ and Shigeo Maeda****
}

\begin{abstract}
In order to investigate the relation between the thickness and the formation of striated subboundaries in the crystals grown from the melt and also to make a direct $\mathrm{X}$-ray observation of dislocations in the similary prepared thin single crystals, a technique has been devised for producing single crystal foils of tin from the melt: After vacuum melting and degassing, molten tin is thrust in a special type glass mould with a pressure of $\mathrm{N}_{2}$ gas. Thus, the production of tin single-crystal foils about $0.007 \mathrm{~cm}$ in thickness is successfully grown from the seed crystal. In this case, carbon is pre-coated on the inner walls of the glass mould to avoid direct contact between tin and glass. For the samples with the purity of $99.999 \%$ or higher and the thickness of less than $0.01 \mathrm{~cm}$, however, this carbon pre-coating technique was ineffective for suppressing of stress induced by the adhesion between glass and tin.

Improvements on the above disadvantages of this technique are now under investigation.
\end{abstract} (Received March 31, 1967)

\section{Introduction}

Two types of substructures have generally been observed in metal crystals grown from the melt ${ }^{(1)}$; one is the impurity substructure, called "corrugation", whose formation is related to a thin zone of "constitutional supercooling" caused by the presence of solute atoms $^{(2)}$; the other is a misoriented substructure, called "striation", subboundaries of which consist of an arrangement of edge-dislocations ${ }^{(3)}$. The former is formed parallel to the direction of the heat flow but its occurrence depends on the three conditions, i.e., high purity, high temperature gradient and low growth rate. On the other hand, an explanation of the formation of the latter was first proposed by Teghtsoonian et al. (3) on the basis of a vacancy mechanism. Later, Frank ${ }^{(4)}$ developed a more detailed model based on the mechanism of collapsing vacancy discs in order to qualitatively explain the origin, size and initial misorientation of the striated substructure. Recently, the mechanism of dislocation-loop-formation based on the vacancy condensation, has qualitatively been examined by Schoeck et al. $^{(5)}$, Elbaum ${ }^{(6)}$ and Jackson ${ }^{(7)}$ : Schoeck et al. suggested that the mechanism proposed by Frank may not be appropriate on the ground that both the number of dislocation loops formed by the collape of vacancy discs and the climb rate of dislocations are insufficient for the formation of the striated subboundaries. Also, Blbaum examined the formation of dislocations by the mechanism

* This paper was presented at the Spring Meeting of the Japan Institute of MetaIs, held on Apr. 1966, Tokyo, Japan.

** Faculty of Science, Kyoto University, Kyoto, Japan.

*** Institute for Chemical Research, Kyoto University, Kyoto, Japan.

***** Kyoto University of Education, Kyoto, Japan.

(1) C. Elbaum : Progr. Met. Phys., 8(1959), 203 ; W.C. Winegard: Metallurgical Rev., 6(1961), 57.

(2) J.W. Rutter, B. Chalmers: Can. J. Phys., 31 (1953), 15.

(3) E. Teghtsoonian, B.Chalmers : ibid., 29 (1951), 370; 30 (1952), 388.

(4) F. C. Frank: Deformation and Flow of Solids, IUTAM Colloquium, Madrid, (1955), 73 .

(5) G. Schoeck, W. A. Tiller : Phil. Mag., 5 (1960), 43.

Trans. J I M of collapsing vacancy discs in aluminium, copper, silicon and germanium, and concluded that this mechanism may hold for metals, but the size of loops is too small to detect by means of optical microscopy in silicon and germanium. Jackson came to a conclusion that the various mechanisms which have so far been suggested on the formation of dislocations cannot fully elucidate the dislocations in melt-grown crystals. Thus, it seems that no mechanism has been established to account for the formation of the striated substructure.

Elbaum $^{(8)}$ has examined the relationship between the dimensions of samples and the dislocation densities on aluminium single crystals in the shape of a carrot by Lang's X-ray method ${ }^{(9)}$, and reported that no dislocation can be observed in the fine part below $0.5 \mathrm{~mm}$ in diameter in the super-pure sample grown under a very carefully controlled condition ${ }^{(10)}$.

In our laboratory, to investigate the relation between the thickness and the formation of striated subboundaries in the crystals grown from the melt on one hand and to carry out the direct $\mathrm{X}$-ray observation of dislocations in thin as-grown single crystals from the melt on the other, a technique was devised for producing single crystal foils of tin from the melt ${ }^{(11)}$. The tin foils grown by this technique, however, showed several cavities and a new boundary was in most cases formed by the resulting mismatch when the crystals grew around the periphery of cavities as shown in Photo. 1*. The as-molten tin was sucked up into a special type glass mould in air, so that the unfilled parts in the mould were stuffed with the molten tin stayed in the foil-part during the crystal growth. In addition, it was impossible for this method

(6) C. Elbaum: ibid., $5(1960), 669$.

(7) K. A. Jackson: ibid., 7 (1962), 1117.

(8) C. Elbaum: J. Appl. Phys., 31 (1960), 1413.

(9) A. R. Lang: ibid., 29 (1958), 597 ; Acta Cryst., 12 (1959), 249.

(10) S. Howe, C. Elbaum: J. Appl. Phys., 32 (1961), 742.

(11) M. Koyama, Y.Tsujii, S. Maeda : Bull. Inst. Chem. Res., Kyoto Univ., 42 (1964), 338.

* An X-ray diffraction micrograph ( $\mathrm{CuK}_{\alpha}$ radiation) of a tin foil with the thickness of $0.015 \mathrm{~cm}$, the purity of $99.8 \%$ and the lowering rate of $0.1 \mathrm{~mm} / \mathrm{min}$. 


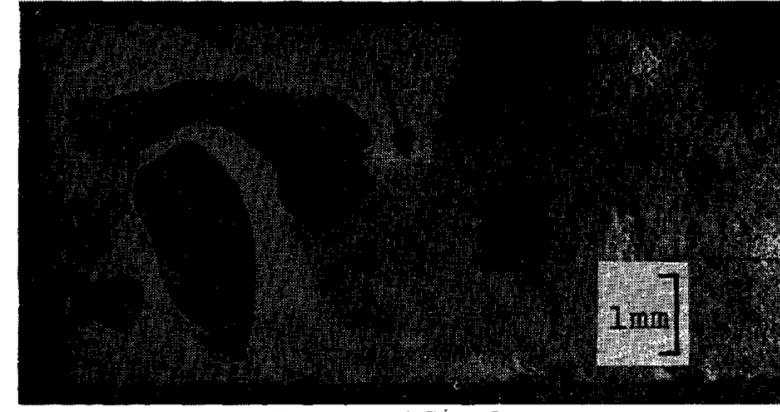

Direction of Growth $\rightarrow$

Photo. 1 Cavities which were formed during growth in the foil produced by sucking up method. A subboundary has newly been formed at the place designated with the arrow.

to produce single crystal foils less than $0.01 \mathrm{~cm}$ in thickness**.

To improve the above disadvantages, a modified technique has been proposed to produce tin single-crystal foils less than $0.01 \mathrm{~cm}$ in thickness ${ }^{(12)}$, and the samples thus prepared have been examined by two X-ray diffraction methods.

\section{Experimental}

\section{Preparation of the glass mould}

Two sheets of glass $\left(1 \times 5 \times 0.14 \mathrm{~cm}^{3}\right.$ in dimensions $)$ were cut down from a photographic plate and two thin sheets of cover glass $\left(0.3 \times 5 \times t \mathrm{~cm}^{3}\right)^{* * * *}$ were also prepared. Then the latter was sandwiched in between the former ${ }^{* * * *}$ in such a way that the longer edges of both sheets were put together as shown in Fig. 1(a), and these longer edges were sealed with an altmina slip mixed

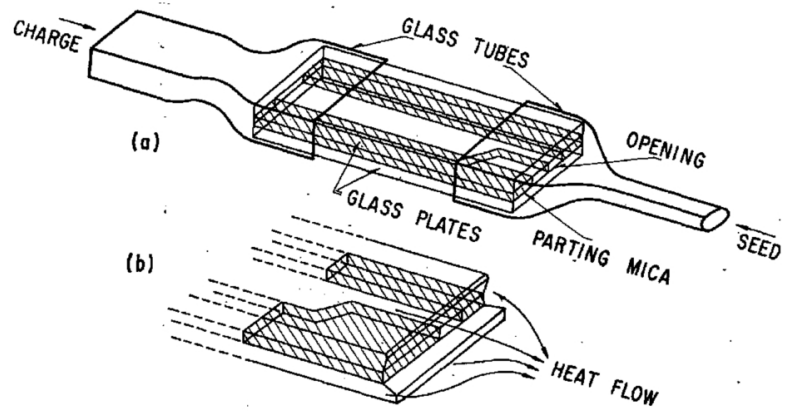

Fig. 1 (a) A special type glass mould used for vacuum casting method. (b) Trimming for annihilating the pre-existing subboundaries in the seed.

** A condition necessary for Lang's X-ray diffraction micrography is that $\mu$ is nearly equal to unity in the following expression :

$$
I=I_{0} \exp (-\mu t)
$$

where $I$ is the intensity after traversing the layer of a matter with the thickness of $t \mathrm{~cm}$, whereas $I_{0}$ reprsents the intensity of the X-rays when $t$ is zero, and the quantity $\mu$ is the "linear absorption coefficient". To realize the condition $\mu t \sim 1, t$ is about $0.008 \mathrm{~cm}$ for $\mathrm{AgK}_{\alpha_{1}}$ radiation in the case of tin.

(12) H. Takaki, M. Koyama, Y.Tsujii, K. Iwauchi, S.Maeda : ibid., 43 (1965), 416.

*** In the case of $t$ less than $0.015 \mathrm{~cm}$, mica plates were used instead of the cover glass.

***** In this case, the dry-plate surfaces coated with photographic emulsion were used as the surfaces necessary to produce a tin foil, and these surfaces were previously coated with silicon oil to prevent the adhesion between these surfaces and the tin foil. with sodium silicate as shown in Photo. 2(a). Then, two glass tubes (boro-silicate glass) were put on both sides of the assembly and the glass mould was also sealed. Then, after pre-heationg the glass mould in vacuum $\left(10^{-5} \mathrm{~mm}\right.$ $\mathrm{Hg}$ ), a single crystal seed with a chosen crystallographic orientation and a charge were inserted, respectively, from both of the open sides as shown in the figure. In this case, the shorter edges of the assembly were trimmed off inwards as shown in Fig.1 (b). By this trimming, the molten charge was smoothly thrust into the opening of the glass mould and the pre-existing subboundaries are almost perfectly annihilated at the foil part. The trimming effect will be much greater when the breadth of the opening is made narrower.

\section{Procedure}

After vacuum melting $\left(10^{-4} \mathrm{mmHg}\right)$, degassing and revolving the apparatus counter-clockwise as shown in Fig. 2, molten tin was pushed into the opening of the

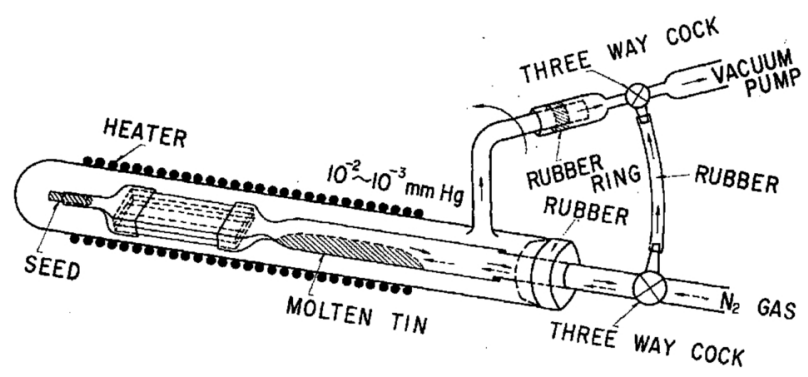

Fig. 2 An apparatus for vacuum melting, degassing and casting with a pressure of $\mathrm{N}_{2}$ gas.

mould with a pressure of $\mathrm{N}_{2}$ gas (99.96\% in purity). It is an essential point in this technique to control a balance between an evacuation degree $\left(10^{-2} \sim 10^{-3} \mathrm{~mm}\right.$ $\mathrm{Hg}$ ) outside the glass mould and a $\mathrm{N}_{2}$ gas pressure inside the mould. The mould thus cast was lowered in a vertical electric furnace with the temperature gradient of $25^{\circ} \mathrm{C} / \mathrm{cm}$ in air. After the crystal growth, the glass mould (Photo. 2 (a)) was soaked in a HF solution and the hard glass tubes, $T_{1}$ and $T_{2}$, were dissolved as shown in Photo. 2 (b). Then the glass plate $P$ was also dissolved after cutting off both ends of the foil part with an acid saw (tin is almost undissolved in HF solution). A macroscopic structure of the tin foils thus exposed (about $0.007 \mathrm{~cm}$ in thickness) is shown in Photo. 3 .

\section{Experimental conditions and observations}

The tin foils examined in the present study were 99.8 to 99.9999 (COMINCO Sn) \% in purity, 0.02 to 0.007 $\mathrm{cm}$ in thickness, and grown in the (001)-[110] orientation at the lowering rates of 2 to $0.07 \mathrm{~mm} / \mathrm{min}$. Furthermore, the perfection of the foils was examined by the Berg-Barrett method and by a transmission type bent-quartz monochromator ( $\mathrm{CuK} \alpha$ radiation $)^{(13)}$.

\section{Results and Discussions}

Photo. 4 shows two sets of Berg-Barrett patterns which

(13) M. Koyama : J. Japan Inst. Metals, 25 (1961), 584. 


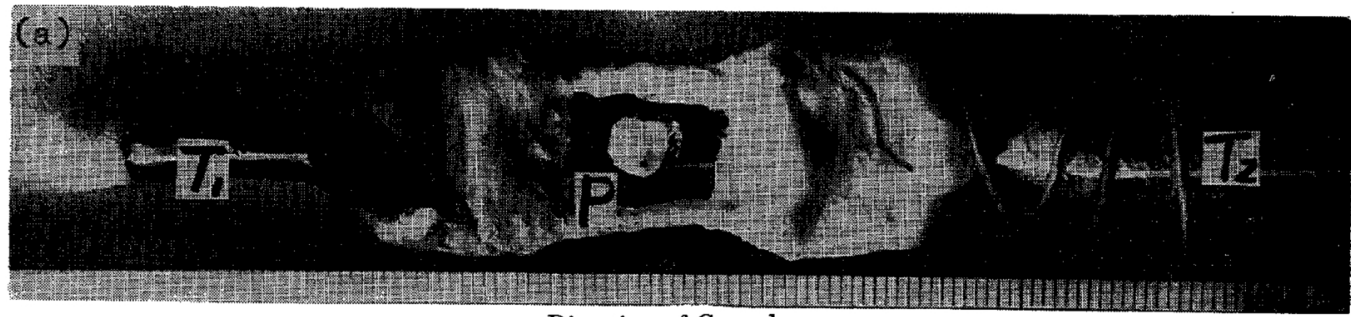

Direction of Growth $\rightarrow$

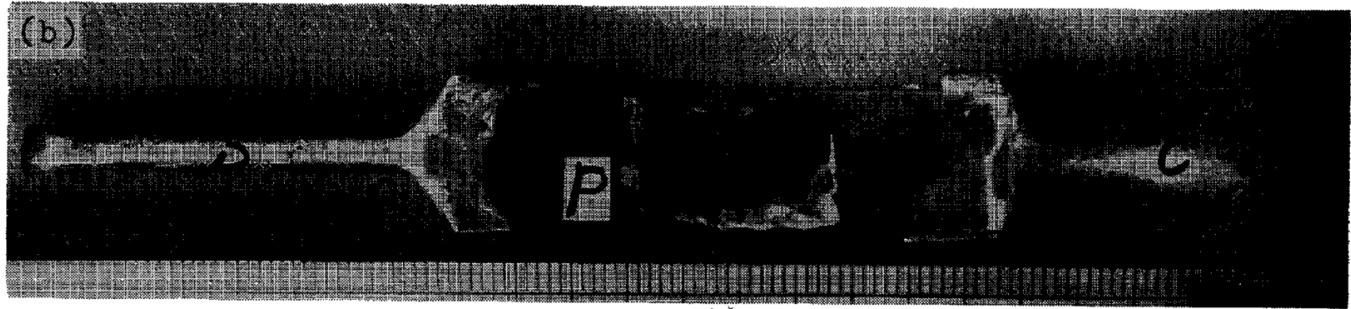

Photo. 2 (a) A special type glass mould after crystal growing. (b) A sample after the glass tubes, $\mathrm{T}_{1}$ and $\mathrm{T}_{2}$ in (a) were dissolved off with a HF solution -P: glass plate, S: seed crystal, C: tin crystal.

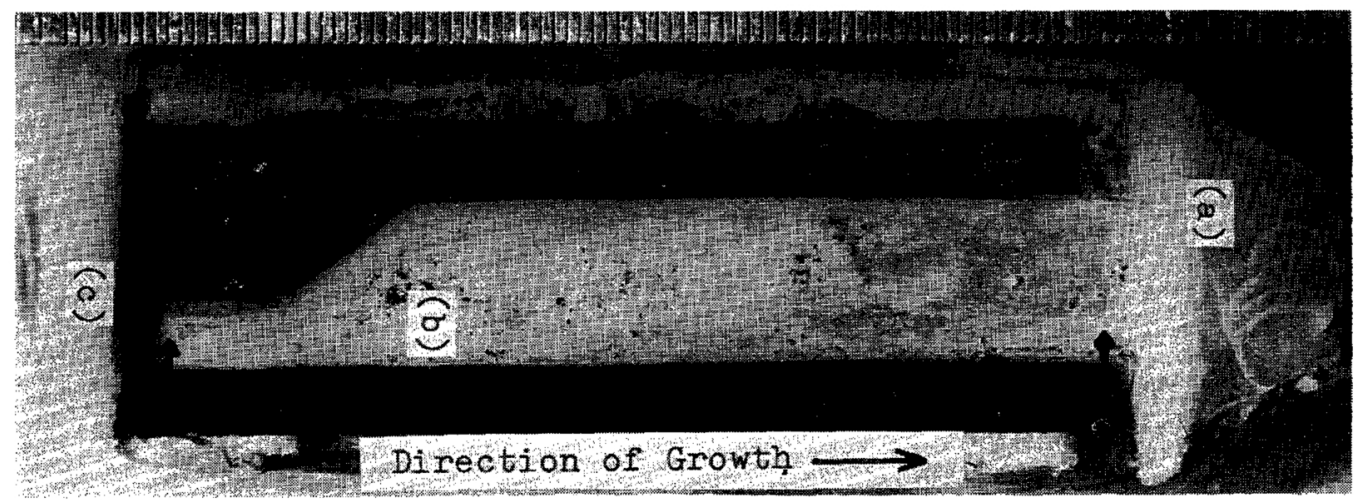

Photo. 3 A macroscopic structure of the tin foil (the part between two short arrows) exposed by treating with a HF solution-the thickness, lowering rate and purity are $0.007 \mathrm{~cm}, 0.31 \mathrm{~mm} / \mathrm{min}$ and $99,999 \%$ respectively. (*In this sample carbon was pre-coated instead of silicon oil as described after).

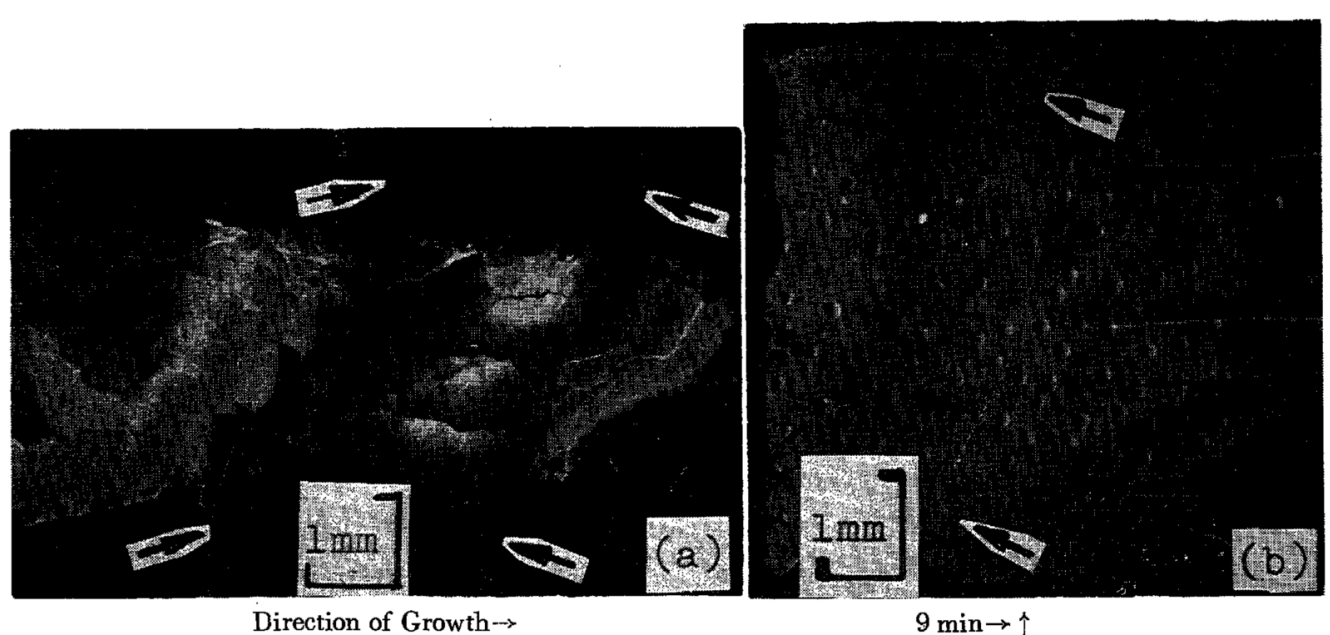

Photo. 4 Two sets of Berg-Barrett photographs which were reflected from the sample $(99.999 \%$ in purity grown at the lowering rate of $0.31 \mathrm{~mm} / \mathrm{min}$. (a) Three pieces of Berg-Barrett photographs from the longitudinal section of the seed part of $4 \mathrm{~mm}$ in diameter. (b) Two pieces of Berg-Barrett photographs from the foil part $0.02 \mathrm{~cm}$ in thickness (incubation period is $9 \mathrm{~min}$ ).

were reflected from the (112) plane* in the crystal $(99.999 \%)$ grown at the lowering rate of $0.31 \mathrm{~mm} / \mathrm{min}$. Three $((\mathrm{a}))$ and two $((\mathrm{b}))$ pieces of Berg-Barrett photographs taken from the different parts on the surface of the sample are combined in this composite photograph. The left-hand photograph, (a), is that from the lon-

* (112) plane was generally used as the reflection plane hereafter. gitudinal section ${ }^{* *}$ of the seed part $4 \mathrm{~mm}$ in diameter, and the right-hand one, (b), is that from the foil part $0.02 \mathrm{~cm}$ in thickness and shows an example of the incubation period prior to the generation of striated subboundaries. The formation of the pitted surface is attributed to the cohesion of pre-coated silicon oil on the

* The longitudinal section was obtained by an acid polishing machine. 
glass plate of the mould during the crystal growth. The wavy lines, running obliquely, which are indicated with a pair of arrows are the joints mentioned above. In (b), the striated subboundaries distinguished by bright or dark striate regions are observed in parallel with each other after an incubation period - these subboundaries have penetrated as deep as the reverse side of the sample, whereas in (a) the irregular subboundaries are observed throughout the section, presumably due to the migration of subboundaries after solidification. In the low-purity samples, however, the straight subboundaries are regularly formed overlapping each other with the impurity subboundaries (corrugations) ${ }^{(1)}$. Photo. 5 shows a Berg-

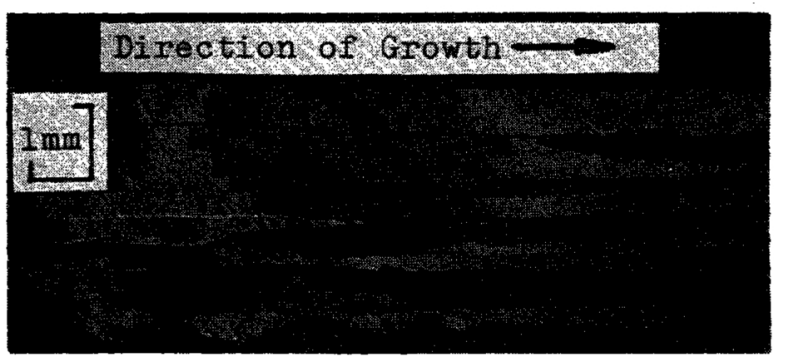

Photo. 5 A Berg-Barrett photograph which was taken from the longitudinal section of the seed part in the sample $(99.8 \%$ in purity) grown at the lowering rate of $0.1 \mathrm{~mm} / \mathrm{min}$. would occur in the foils with the thickness of less than $0.14 \mathrm{~cm}$ according to Frank's theory ${ }^{(4)}: t$ is less than the square root of the product of diffusion coefficient and diffusion time. Even in the sample $0.01 \mathrm{~cm}$ in thickness, however, the striated subboundaries with a spacing of $0.07 \mathrm{~cm}$ were observed, and the dislocation density of the sample was larger than that in the $0.02 \mathrm{~cm}$ thick sample. Furthermore, the spacing between the striated subboundaries in the sample of $99.8 \%$ purity was wider than in that of $99.999 \%$ purity as in the case of impurity subboundaries.

The effect of stress, induced by an adhesion between the sample surface and the inner wall of the glass mould, seems to be an important clue to explain these experimental results, and this effect seems to be stronger with increasing purity. For example, Photo. 6 (a) shows a macro-structure of the longitudinal section ${ }^{* *}$ of the part C (see Photo. 2 (b)) of the sample with the purity of $99.9999 \%$ and the lowering rate of $0.1 \mathrm{~mm} / \mathrm{min}$. The bright region surrounding the figure may be that effected by the adhesion mentioned above. Photo. 6 (b) shows the Berg-Barrett patterns which were taken from the parts designated with the arrows, 1,2 and 3 respectively in the crystal part between two subboundaries, $A-B$ and $C-D$ as sketched for reference. The reflected images are wider at the part 2 near the periphery than
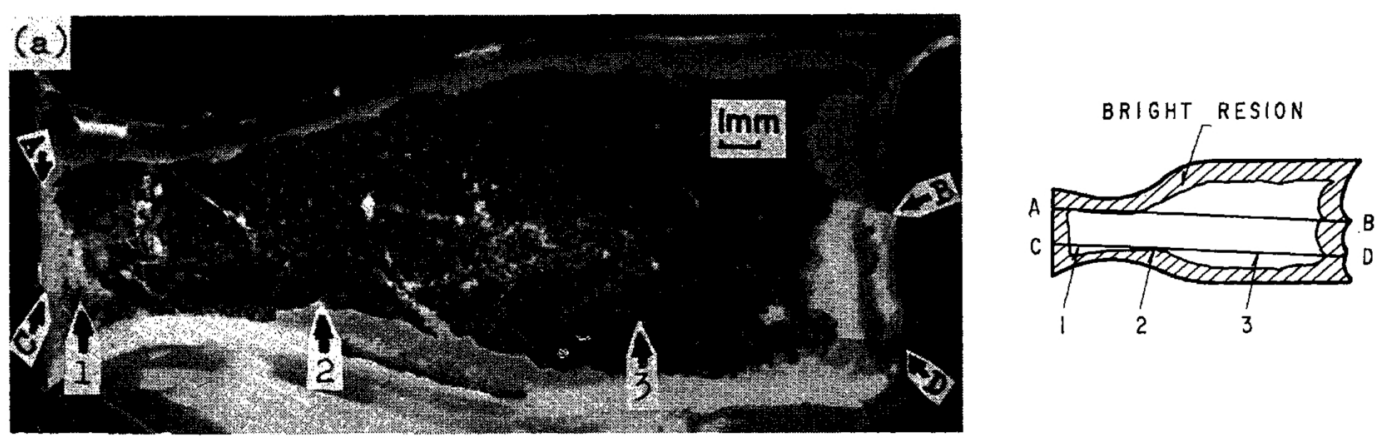

Direction of Growth $\rightarrow$

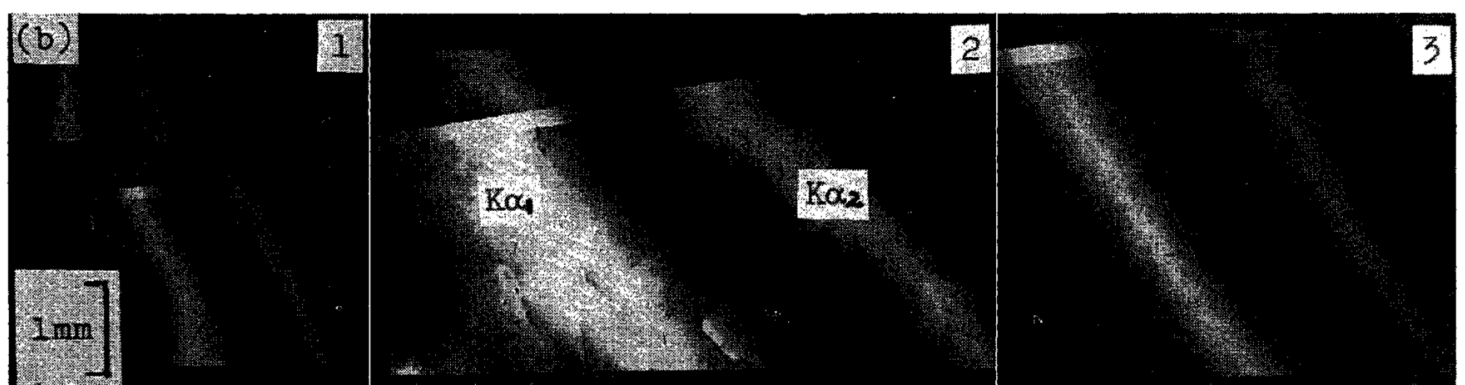

Photo. 6 (a) A macro-structure of the longitudinal section of the last part to freeze in the sample, in which purity and lowering rate are $99.9999 \%$ and $0.1 \mathrm{~mm} / \mathrm{min}$. (b) Three pieces of Berg-Barrett photographs which were taken from the three parts designated with the arrows, 1,2 and 3 in (a). The images were reflected from the crystal part between two subboundaries, A-B and C-D (see the sketch beside (a)).

Barrett photograph from the longitudinal section of the seed part $(99.8 \%, 0.1 \mathrm{~mm} / \mathrm{min}) * *$.

Furthermore, the sample $0.02 \mathrm{~cm}$ in thickness, for example, showed a longer incubation period than the $0.01 \mathrm{~cm}$-thick sample, its misorientation of subboundaries increased less steeply, and a wider spacing between the subboundaries was observed.

If the super-saturated vacancies should diffuse out from the flat surfaces of foils, no dislocations at the parts 1 and 3 . The bright regions at the upper and lower sides in (a), therefore, seem to be related to the adhesion mentioned above.

Accordingly, the inner walls of glass, $T_{1}$ and $P$, in Photo. 2 (a) were pre-coated with carbon before vacuum casting to avoid direct contact between tin and glass. For example, Photo. 7 shows two pieces of Berg-Barrett photographs which were taken by reflection from the etched surface of the foil grown in a carbon-coated glass 


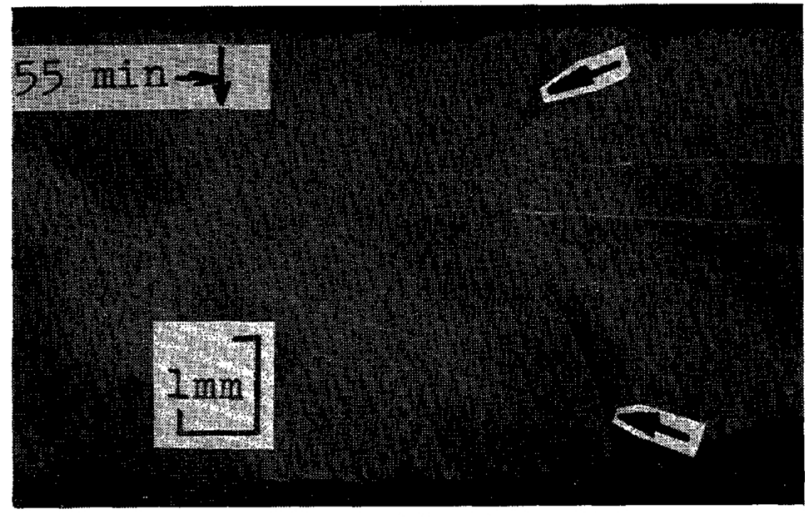

Direction of Growth $\rightarrow$

Photo. 7 Two pieces of Berg-Barrett photographs which were reflected from the etched surface of the foil grown in the same condition as in the foil of Photo. 4 (b) except using a carbon-coated glass mould (incubation period is $55 \mathrm{~min}$ ).

mould. In this case, the growth condition was the same as in the sample of Photo. $4(\mathrm{~b})$. The generation of striated subboundaries is very slight and the incubation period is very long (about $55 \mathrm{~min}$ ) as compared with that in Photo. 4 (b) (about $9 \mathrm{~min}$ ). For the samples with the purity of $99.999 \%$ or more and the thickness of less than $0.01 \mathrm{~cm}$, however, the above carbon pre-coating technique is ineffective as described below. Photo. 8 shows
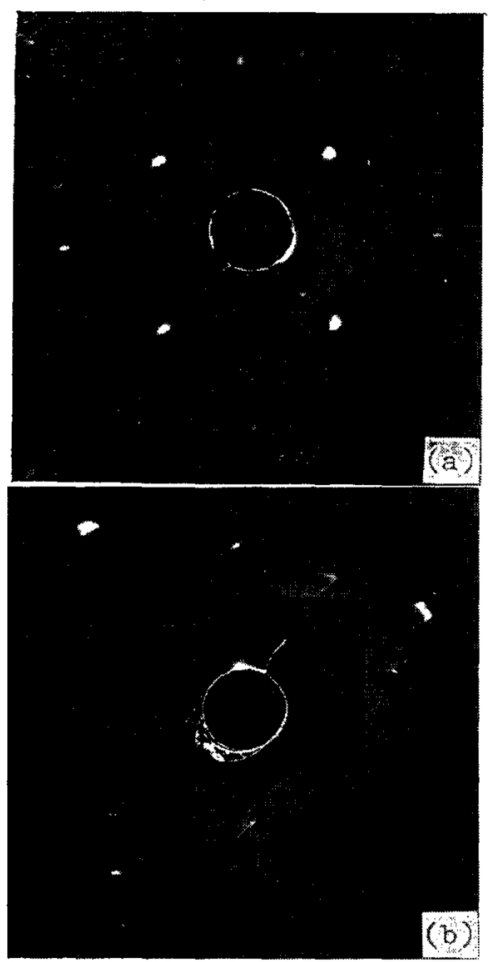

Photo. 8 Two back reflection Laue diagrams which were obtained from two foils grown in the same condition $(99.999 \%, 1.18 \mathrm{~mm} / \mathrm{min}$ and carbon-coating) except the thickness: (a) $0.015 \mathrm{~cm}$; (b) $0.007 \mathrm{~cm}$.

an example of the ineffectiveness of carbon pre-coating. These two back reflection Laue patterns (the distance from sample to film is $3 \mathrm{~cm}$ hereafter) are those obtained from the single crystal foils of 0.015 (in (a)) and $0.007 \mathrm{~cm}$ (in (b)) in thickness, respectively. These two foils were grown under the same condition $(99.999 \%$,

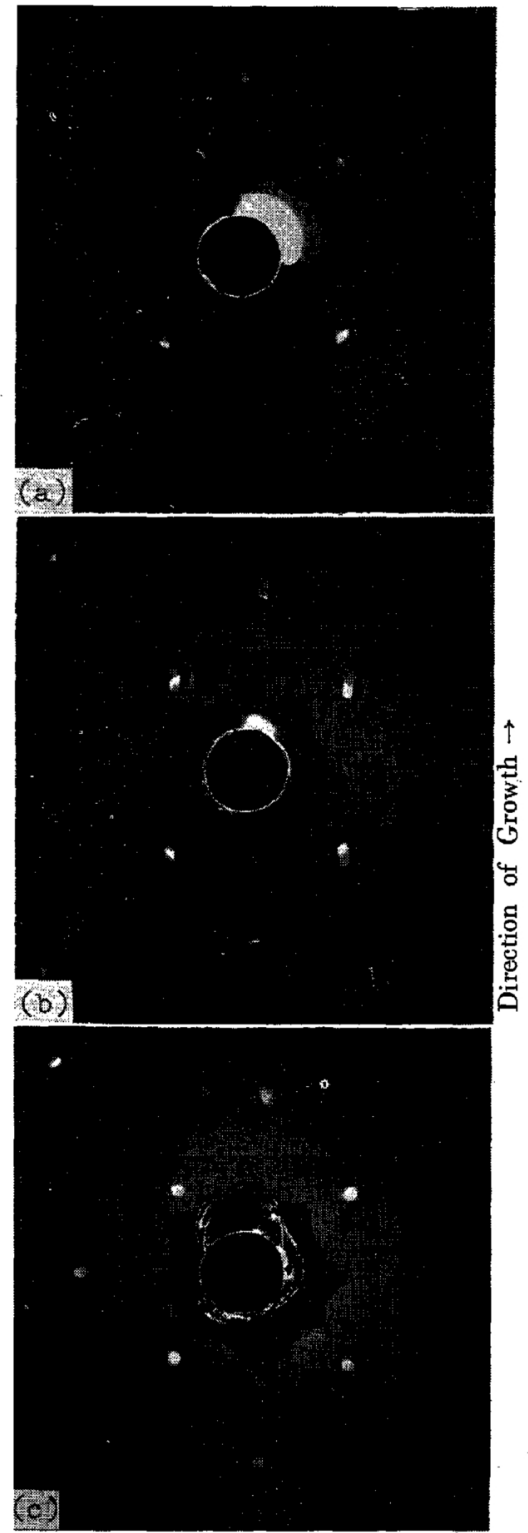

Photo. 9 Three back reflection Laue diagrams which were obtained from the three parts, (a), (b) and (c), on the sample in Photo. 3 (carboncoating). (a) and (c) are from the upper and lower bulk crystal parts, respectively. (b) is from the foil part.

$1.18 \mathrm{~mm} / \mathrm{min}$ and carbon-coating) except the thickness. Another example is given in Photo. 9, in which are shown three back reflection Laue patterns from the three parts of the sample in Photo. 3. In this sample, the thickness, the lowering rate and the purity were 0.007 $\mathrm{cm}, 0.31 \mathrm{~mm} / \mathrm{min}$ and $99.999 \%$, respectively, and the carbon pre-coating technique was used. Photo. 9 (a) and (c) were obtained from the upper and lower bulk crystal parts of the sample, respectively, and Photo. 9 (b) was taken from the foil part. The individual diffraction spots appear as asterism in the Laue diagram obtained from the foil part. Photo. 10 shows a macro-structure of the sample $0.007 \mathrm{~cm}$ in thickness, from which the Laue diagram in Photo.8 (b) was obtained****. The socalled striated substructure is observed after the foil-

*** This sample was grown in an undesired orientation because of mis-melting of the seed at the start of growth, and was chemically etched. 


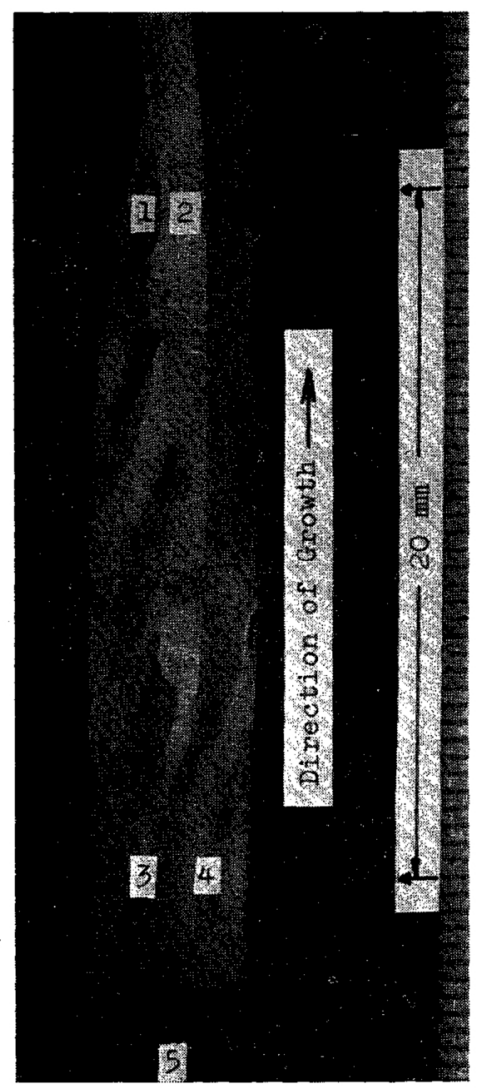

Photo. 10 A macro-structure of the foil $0.007 \mathrm{~cm}$ in thickness, from which was obtained Photo. 8 (b).

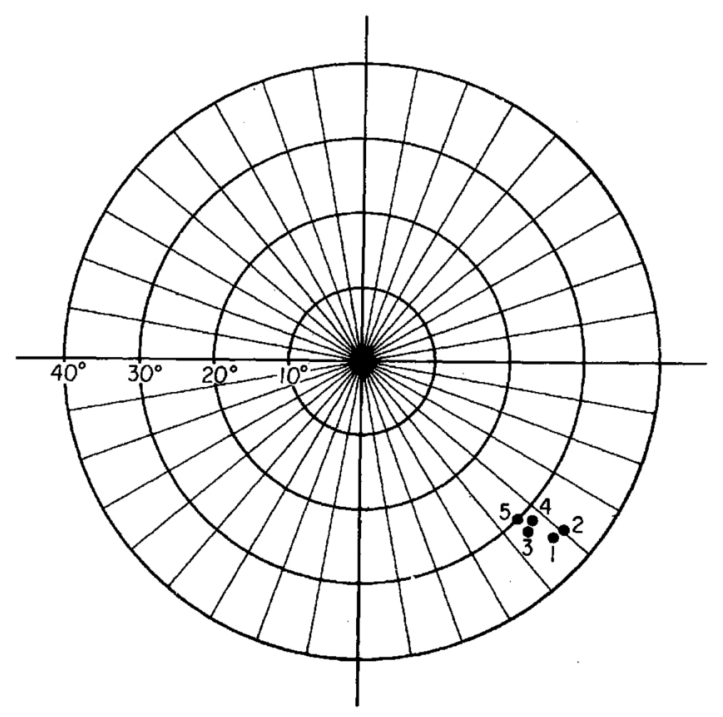

Fig. 3 A stereo-diagram of each [110] orientation of the parts designated with the numerals, $1,2,3,4$ and 5 , in Photo. 10. The center of the diagram represents the direction of the longitudinal axis of the foil, and the part 5 is a part of the bulk crystal.

growth of about $5 \mathrm{~mm}$. Fig. 3 shows a stereo-diagram of each [110] orientation of the parts designated with the numerals, 1, 2, 3, 4, and 5, in Fhoto. 10. The center of the diagram, however, represents the direction of the longitudinal axis of the sample, and the part 5 is relative to a part of the bulk crystal. The figure indicates that the foil crystal is twisted by the (101)-[10I] slip-system at a rate of about 2 degrees of arc per $\mathrm{cm}$ in the course of growing. This phenomenon seems to be interpreted
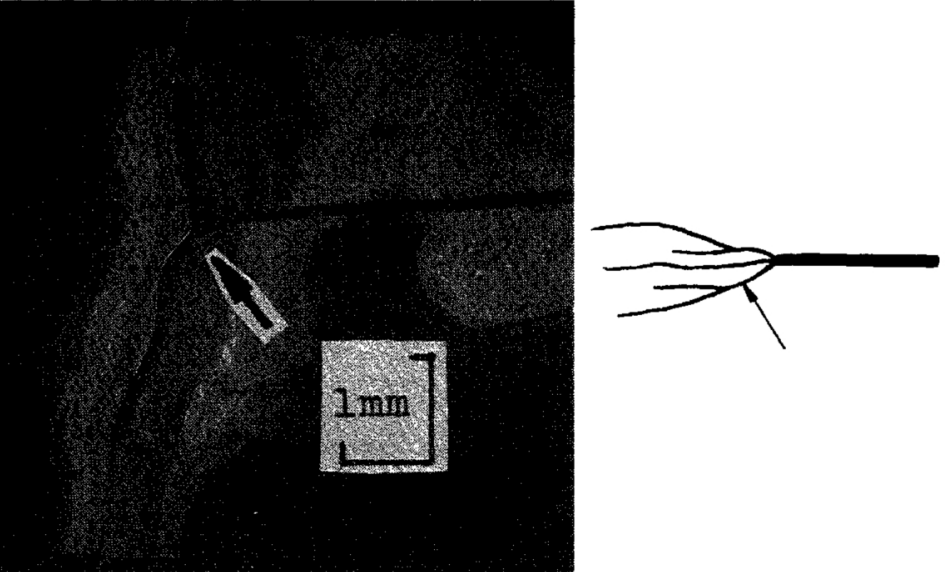

Direction of Growth $\rightarrow$

Photo. 1.1 Three pieces of Berg-Barrett photographs from the foil, from which was obtained Photo. 8 (a), showing an example of the "river-type substructure".

as follows: The part already frozen is subjected to plastic-deformation while, growing, under a stress induced by an adhesion between glass wall and tin ${ }^{(14)}$.

Three pieces of Berg-Barrett photographs are given in Photo. 11 which shows an example of the "river-type substructure"(15) in a sample whose purity, lowering rate and thickness are $99.999 \%, 1.18 \mathrm{~mm} / \mathrm{min}$ and 0.015 $\mathrm{cm}$, respectively, as already explained in Photo. 8(a)****. It seems that this structure appeared at the part where the pre-coated carbon film was peeled away by chance. As explained by Chalmers, it is not clear why dislociations of the same sign aggregate together when dislocations of the different sign could annihilate each other, or why similar low-angle subboundaries join together instead of annihilating the oppositely tilted subboundaries. In the present study, it is assumed that the multiplication of new dislocations from the already existing virgin ones or their movements could be caused by stress induced between glass wall and tin. The creation of virgin dislocations, however, is ambiguous. These dislocations may result from either the homogeneous nucleation of vacancy clusters or the heterogeneous nucleation by impurity particles as assumed by Jackson ${ }^{(7)}$ and Evans et al. ${ }^{(17)}$. There is another possibility that they result from both of the mechanisms mentioned above.

Images of individual dislocations are generally revealed by Lang's method only when the density is less than $10^{6}$ per $\mathrm{cm}^{2}$. So, it is desired in this study that single crystal foils of $10^{5}$ per $\mathrm{cm}^{2}$ or less in dislocation density be prepared. An indirect method has been known to derive an approximate dislocation density from the width of X-ray diffraction lines. It has been said, however, that this method is unreliable when the dislocation density is less than $10^{4}$ per $\mathrm{cm}^{2}$. As mentioned already, the single crystal foils of tin thus prepared may be fairly imperfect. So, a relatively perfect foil

(14) S. Takeuchi, H. Suzuki and S. Ikeda: 38th Meeting Japan Inst. Metals, Pre-print (1956), 17.

(15) B. Chalmers: Principles of Solidification, (1964), 60.

****** It has been shown by Chalmers et al. that the lineage structure (striations) in aluminium originates as a river-type structure(16).

(16) P. E. Goherty, B. Chalmers: Trans. AIME, 224 (1962), 1124.

(17) K. R. Evans, W. F. Flanagan : Phil. Mag., 14 (1966), 1131. 


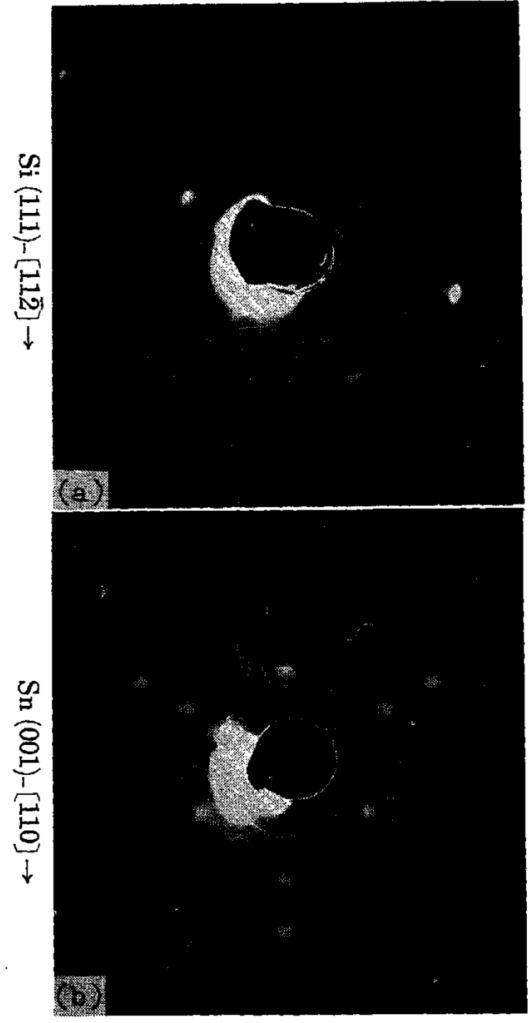

Photo. 12 Two back reflection Laue diagrams obtained from the tin and silicon single crystals. (a) Tin foil: purity, $99.9999 \%$; lowering rate, $0.1 \mathrm{~mm} / \mathrm{min}$; thickness, $0.014 \mathrm{~cm}$; carboncoating. (b) Silicon single crystal : dislocation density, $10^{2}$ per $\mathrm{cm}^{2}$.

whose purity, lowering rate and thickness were 99.9999 $\%, 0.1 \mathrm{~mm} / \mathrm{min}$ and $0.014 \mathrm{~cm}$, respectively, was examined by an X-rays technique in comparison with a silicon single crystal with the dislocation density of $10^{2}$ per $\mathbf{c m}^{2}$. Photo. 12 shows two back reflection Laue diagrams which were obtained from these crystals. There is almost no discrepancy in the degree of perfection between the two crystals from the images of Laue spots. A considerable discrepancy in the degree of perfection, however, is observed in Photo. 13 . which shows the images diffracted from the crystals by a transmission type bent-quartz monochromator. In this case, the reflection images in (a)***** and (b) are those from (112) in tin and from (111) in silicon, while the glancing angles are 11 and 15 degrees of arc and the Bragg angles are 31.37 and 14.23 degrees of arc, respectively - the distances from the target to the monochromator, the monochromator to the sample, and the sample to the film are 16,10 , and $30 \mathrm{~cm}$, respectively.

An adhesion between glass and molten tin seems to be so feeble in a reduced atmosphere that molten tin is generally used in the preparation of float glass plates. In the present case, however, photographic plates (soda glass) are contacted with molten tin (about $300^{\circ} \mathrm{C}$ ) in air during crystal growth. As a result, the bonding of molten

****** The wavy images are attributed to the unevenness of the foil surface caused by chemical etching.

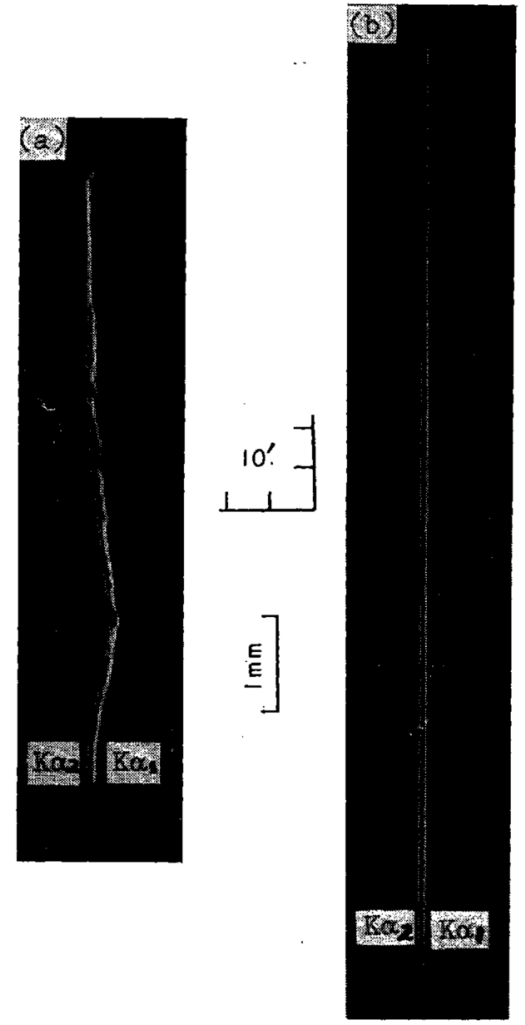

Photo. 13 Two reflection images which were obtained from the samples explained in Photo. 12 by using a transmission type bent-quartz monochromator. (a) An image reflected from (112) in the tin foil. (b) An image reflected from (111) in the silicon crystal.

tin and glass may be realized by sodium or oxygen ions. If the crystal is grown in vacuum, molten tin may be sucked off from the foil part, while the absorption of gas into molten tin may be caused during the crystal growth in a reduced atmosphere. As mentioned already, therefore, photographic plates were pre-coated with carbon which was generated by imperfect combustion of town gas. In case the thickness of the foil is less than $0.01 \mathrm{~cm}$, however, the adhesion between glass and molten tin seems to be especially strong at a high purity of tin. As the ratio of the surface area to the volume increases hyperbolically with decreasing thickness of the foil, phenomena relative to the surface are expected to be effected increasingly as the foil thickness is decreased. It is assumed, therefore, that the carbon film coated on soda glass seems to be insufficient to prevent the adhesion mentioned above. To improve such a disadvantage, it may be effective to use vycor or silica glass instead of soda glass and to deposit carbon in vacuum, or it may be more desirable to use glassy carbon plates rather than glass and to pre-coat with either silicon oil or carbon.

At present, it may be possible to produce tin single crystal foils which enable the direct observation of dislocations, if the thickness, $t$ is increased to $0.015 \mathrm{~cm}$ in spite of $\mu t$ larger than unity. 